\title{
DNA methylation changes in ovarian cancer are cumulative with disease progression and identify tumor stage George S Watts*1,3, Bernard W Futscher ${ }^{2,3}$, Nicholas Holtan ${ }^{3}$, Koen DeGeest ${ }^{4}$, Frederick E Domann ${ }^{5}$ and Stephen L Rose $e^{4,6}$
}

\author{
Address: ${ }^{1}$ Department of Medical Pharmacology, College of Medicine, University of Arizona, Tucson AZ 85724 USA, ${ }^{2}$ Department of \\ Pharmacology and Toxicology, College of Pharmacy, University of Arizona, Tucson AZ 85724 USA, ${ }^{3}$ Arizona Cancer Center, University of Arizona, \\ Tucson AZ 85724 USA, ${ }^{4}$ Department of Obstetrics and Gynecology, Free Radical \& Radiation Biology Program, University of Iowa, Iowa City IA \\ 52242 USA, 5 Department of Radiation Oncology, Free Radical \& Radiation Biology Program, University of Iowa, Iowa City IA 52242 USA and \\ ${ }^{6}$ Department of Obstetrics and Gynecology, University of Wisconsin, Madison WI, 53792, USA \\ Email: George S Watts* - gwatts@azcc.arizona.edu; Bernard W Futscher - bfutscher@azcc.arizona.edu; \\ Nicholas Holtan - nholtan@email.arizona.edu; Koen DeGeest - koen-degeest@uiowa.edu; Frederick E Domann - frederick- \\ domann@uiowa.edu; Stephen L Rose - srose2@wisc.edu \\ * Corresponding author
}

Published: 30 September 2008

BMC Medical Genomics 2008, I:47 doi:10.1 186/1755-8794-1-47
Received: 18 December 2007

Accepted: 30 September 2008

This article is available from: http://www.biomedcentral.com/1755-8794/1/47

(C) 2008 Watts et al; licensee BioMed Central Ltd.

This is an Open Access article distributed under the terms of the Creative Commons Attribution License (http://creativecommons.org/licenses/by/2.0), which permits unrestricted use, distribution, and reproduction in any medium, provided the original work is properly cited.

\begin{abstract}
Background: Hypermethylation of promoter CPG islands with associated loss of gene expression, and hypomethylation of $C_{p} G$-rich repetitive elements that may destabilize the genome are common events in most, if not all, epithelial cancers.

Methods: The methylation of 6,502 CpG-rich sequences spanning the genome was analyzed in 137 ovarian samples (ten normal, 23 low malignant potential, I 8 stage I, 16 stage II, 54 stage III, and I6 stage IV) ranging from normal tissue through to stage IV cancer using a sequence-validated human $C_{p} G$ island microarray. The microarray contained 5' promoter-associated $C_{p} G$ islands as well as $C_{p} G$-rich satellite and Alu repetitive elements.

Results: Results showed a progressive de-evolution of normal CPG methylation patterns with disease progression; 659 CpG islands showed significant loss or gain of methylation. Satellite and Alu sequences were primarily associated with loss of methylation, while promoter $C_{p} G$ islands composed the majority of sequences with gains in methylation. Since the majority of ovarian tumors are late stage when diagnosed, we tested whether DNA methylation profiles could differentiate between normal and low malignant potential (LMP) compared to stage III ovarian samples. We developed a class predictor consisting of three CPG-rich sequences that was $100 \%$ sensitive and $89 \%$ specific when used to predict an independent set of normal and LMP samples versus stage III samples. Bisulfite sequencing confirmed the NKX-2-3 promoter $C_{P G}$ island was hypermethylated with disease progression. In addition, 5-aza-2'-deoxycytidine treatment of the ES2 and OVCAR ovarian cancer cell lines re-expressed NKX-2-3. Finally, we merged our CPG methylation results with previously published ovarian expression microarray data and identified correlated expression changes.
\end{abstract}

Conclusion: Our results show that changes in $C_{P G}$ methylation are cumulative with ovarian cancer progression in a sequence-type dependent manner, and that $C_{p} G$ island microarrays can rapidly discover novel genes affected by $C_{p} G$ methylation in clinical samples of ovarian cancer. 


\section{Background}

Ovarian cancer remains the most deadly gynecologic malignancy. There were an estimated 22,430 new cases and 15,280 deaths in the United States in 2007[1]. In contrast to other gynecologic malignancies, $81 \%$ of ovarian cancers are late stage III or IV at the time of diagnosis, implying upper abdominal or distant metastases[1]. Because of late stage at diagnosis, the 5 year survival of ovarian cancer for all stages is only $45 \%$, and the 5 year survival for patients with stage III or IV disease is only $30 \%[1]$.

Aberrant DNA methylation at $\mathrm{CpG}$ islands, often in close proximity to transcription start sites, is associated with the epigenetic regulation of genes through altered transcription factor binding and chromatin structure[2]. Widespread changes in CpG island methylation have also been associated with neoplastic progression [3-5]. Previous researchers have successfully used various genome-wide scanning approaches to analyze tumor cells and their epigenomic states [6-10]. Results have shown that CpG islands display tumor-specific patterns of aberrant methylation[6], while selected CpG islands have been reported to show stage-specific patterns of aberrant methylation [11-15], and others have not[16]. Two recently published studies have examined broad epigenetic changes in ovarian cancer[10,11]. Widschwendter et al. examined DNA hypomethylation at satellite sequences and found a strong association between satellite DNA hypomethylation and advanced tumor stage and grade. In addition, satellite DNA hypomethylation was found to be an independent marker of prognosis. Wei et al. used CpGisland microarrays to analyze stage III and IV ovarian tumor samples and developed a classifier that predicted six month progression free survival with 95\% accuracy and specificity. Neither of these studies performed a study of CpG island methylation across all stages of ovarian cancer as reported here.

We have used a 6,560 element CpG island microarray to analyze the epigenomic profile of clinical samples of ovarian tissues ranging from benign to late stage tumors. We identified CpG methylation changes that progressed with disease severity and distinguished stage III ovarian cancer from normal or LMP ovarian tissue. We confirmed the microarray results with bisulfite sequencing, 5-aza-2'deoxyazacytidine re-expression, and correlation with previously published expression profile data.

\section{Methods}

\section{Sample Collection and Nucleic Acid Isolation}

Ovarian tissue samples were obtained from The University of Iowa Gynecologic Oncology Tumor Bank. Samples were collected with informed consent in accordance with the standards of the Institutional Human Subjects Protec- tion Review Board and were surgically staged according to FIGO staging guidelines. Tumor samples were taken from primary tumors only, with no prior exposure to chemotherapy. Normal tissue and tissue samples of low malignant potential were also collected for comparison to tumor. Tissues were snap frozen at the time of surgery in liquid nitrogen and high molecular weight tumor DNA extracted with Trizol reagent by following the manufacturer's instructions (Life Technologies, Inc., Gaithersburg, Maryland). The DOT quantitative test was used to estimate the DNA concentration[17].

\section{CpG island library, probe preparation, microarray production}

The CpG island clones, their preparation for printing, and microarray production were performed as previously described[18].

\section{Target preparation}

Genomic DNA was cut be MseI (New England Biolabs, Beverly, MA), and then a catch-linker was ligated to the MseI fragments. The fragments were then cut with a methylation-specific restriction enzyme, McrBc (New England Biolabs, Beverly, MA). Mock-cut reference samples were exposed to the same conditions and reagents as the digested samples; however, no GTP was added to drive the restriction digest. Twenty nanograms of the mockdigested or twenty nanograms McrBc-cut genomic DNA was then amplified by PCR using primers specific to the linkers and purified with the QIAquick PCR purification kit (Qiagen, Valencia, CA). Fluorescent Cy3 or Cy5 dye was incorporated into the PCR product using the BioPrime DNA labeling system (Invitrogen, Carlsbad, CA).

\section{CpG Island Microarray}

Two-color fluorescence hybridizations analogous to expression microarrays were used to compare DNA digested with the methylation-specific enzyme McrBc to mock-digested reference DNA as described previously[18]. After labeling, cut and mock reactions are mixed and re-purified with the QIAquick PCR purification kit. After purification, the labeled target was lyophilized to dryness, re-suspended in 60 microliters Oligo Hyb Buffer (The Gel Company, San Francisco, CA) and denatured by boiling for ten minutes. The sample was then added to the processed array slide in a chamber of the ArrayBooster Hybridization Station (Advalytix, Concord, MA). An AdvaCard with two mixer chips (Advalytix, Concord, MA) was used to cover and seal the array during hybridization at $42{ }^{\circ} \mathrm{C}$ for $4-8$ hours. Following hybridization, slides were washed by placing them into $50 \mathrm{ml}$ conical tubes containing $2 \times$ SSC, $0.1 \%$ SDS for five minutes, $0.06 \times$ SSC, $0.1 \%$ SDS for five minutes, and $0.06 \times$ SSC for five minutes all at room temperature. Slides were dried by centrifugation at $500 \times \mathrm{g}$ for one minute and scanned for Cy3 
and Cy5 fluorescence using an Axon GenePix 4000 (Axon Instruments, Foster City, CA).

\section{Data Analysis}

The data from scanned microarray images were extracted using GenePix software. Median pixel intensity of each spot was used for analysis. To normalize $\mathrm{Cy} 3$ and $\mathrm{Cy} 5$ signal intensities we used the "interactive linear regression" approach with minor modifications[19]. Raw data was normalized using unmethylated mitochondrial[20] sequences dispersed across the microarrays: first, all intensity values were log transformed, than linear regression was performed using data from the mitochondrial sequences only. Residuals were calculated and outliers (those residuals where $|e|>2 \times$ standard deviation of e) were removed and the regression function was recalculated. If the difference between the r-squared values of the new and previous regression line was less than 0.001 , then no further residuals were removed. Y-Intercept values were applied as correction factors to the log transformed channel 2 values of all clones. The result is that the function of log channel 1 and log channel 2 of mitochondrial clones closely approximates $\mathrm{y}=\mathrm{x}$. Data was loaded into BRB ArrayTools v3.5.0 for normalization between arrays and analysis. Representative $M$ versus A plots for three hybridizations are shown in Additional File 1. Samples were labeled as normal, LMP, or stage III for class prediction using the Prediction Analysis of Microarray (PAM) classification algorithm, with separate training and test sample sets as recommended by Simon et al. [21]. The PAM algorithm as implemented in BRB ArrayTools was used to create linear combinations of loci and weight them according to the stability of their methylation profile within each group of samples (normal/LMP or stage III). The PAM algorithm then attempted to identify the smallest list of loci with the highest prediction accuracy as measured by 10 -fold cross-validation.

Class prediction using five other methods was performed in BRB ArrayTools using sequences univariately significant at $\mathrm{p}<0.001$. Only data from the training set was used to develop the classifier. Cross-validation was done using the leave-one-out method and repeating the entire analysis for each iteration of the cross-validation including determination of which sequences were univariately significant. The permutation p-value for the cross-validated mis-classification rate was estimated by analysis of 2000 permutations in which the sample labels were scrambled and the class prediction analysis performed. The permutated p-value is the proportion of the random permutations that gave as small a cross-validated misclassification rate as was obtained with the real class labels. Additional information on how BRB ArrayTools implements the algorithms and performs cross-validation can be found in the manual: http://linus.nci.nih.gov/pilot/Manual.doc. All other settings were default.

\section{NKX2-3 Bisulfite sequencing}

Five micrograms of genomic DNA was modified with sodium bisulfite as previously described[22]. The NKX2-3 CpG islands were amplified from the bisulfite-modified DNA by two rounds of PCR using nested primers: Forward Primer 1: 5'-GTGGTTTTGATGATGTTATTAA-3', Reverse Primer 1: 5'-ACTCCCTTACAAATACCTAC-3', Forward Primer 2: 5'-AGTTAAAGATATTTTGAATTTGGA-3', Reverse Primer 2: 5'-CTAACAAAATCTATAAACTATTTAT-3'. Both rounds of PCR were performed under the same parameters, with $1 \%$ of the first round PCR product serving as the template in the second round of PCR. PCR amplification was performed in an MJ thermal cycler (PTC200) under the following conditions: $94 \mathrm{C}$ for four min followed by five cycles of $94 \mathrm{C}$ for one min, $56 \mathrm{C}$ for two $\mathrm{min}, 72 \mathrm{C}$ for three min, then 35 cycles of $94 \mathrm{C}$ for $30 \mathrm{~s}, 56 \mathrm{C}$ for two $\mathrm{min}, 72 \mathrm{C}$ for $1.5 \mathrm{~min}$, and a final extension of $72 \mathrm{C}$ for six $\min$.

The resulting PCR product was cloned into a TA cloning vector according to the manufacturer's instructions (pGEM-T-Easy cloning kit, Promega, Madison, WI). Fortyseven positive recombinants from each of ten samples (five normal ovaries, and 5 stage III or IV ovarian tumors) were isolated using a Qiaprep Spin Plasmid Miniprep kit (Qiagen, Valencia, CA) according to the manufacturer's instructions and sequenced on an ABI automated DNA sequencer by the Genomic Analysis, Technology, \& Sequencing Core at the University of Arizona. The methylation status of individual $\mathrm{CpG}$ sites was determined by comparison of the sequence obtained with the known target sequence. The number of methylated CpGs in each sample was counted and averaged over each tissue class. The means for normal and tumor tissue were compared using an unpaired Welch's t-test.

\section{Cell culture and drug treatment}

ES2 cells were maintained in McCoy's media at $37 \mathrm{C}$ under $95 \% / 5 \%$ air/ $\mathrm{CO}_{2}$ atmosphere with $10 \%$ fetal bovine serum and $100 \mathrm{mg} / \mathrm{mL}$ penicillin and streptomycin. OvCar 3 cells were maintained in the same conditions as ES3 except the media was RPMI 1640. 5-aza-2'-deoxycytidine (Sigma-Alrich, St. Louis, MO) was added to the culture media at a concentration of 0,2 , or ten micromolar for six days with drug added every other day starting on day one. On day six cells were washed with $4 \mathrm{C}$ phosphate buffered saline and collected for RNA isolation using Qiagen's RNeasy Midi kit according to manufacturer's instructions. 


\section{ENKX2-3 Real Time RT-PCR}

PCR amplification was performed using Taqman primer/ probes specific for NKX2-3 obtained from Applied Biosystems (Foster City, CA); the primer probe sequences are available upon request. PCR was performed with the ABI Prism 7000 sequence detection system following Applied Biosystem's PCR Master Mix protocol. Real-time PCR was carried out in triplicate on five nanograms of cDNA using parameters recommended by Applied Biosystems. Relative expression was determined by applying the comparative $\mathrm{C}_{\mathrm{t}}$ method, as described previously[23].

\section{Results \\ DNA methylation differences in benign and malignant ovarian tissue}

137 samples of benign and malignant ovarian tissues (ten normal, 23 low malignant potential, 18 stage I, 16 stage II, 54 stage III, and 16 stage IV) from various stages and histopathologies were analyzed to develop a broad view of DNA methylation changes with progression of ovarian cancer (Additional File 2). The CpG island microarray contained 6,560 CpG-rich genomic clones, representing approximately $15 \%$ of the CpG islands in the genome[24]. The CpG islands spanned the genome with varying proximity to 5 ' promoter regions: rank 1 were within $0.5 \mathrm{~kb}$ of transcription start, rank 2 were within 1 $\mathrm{kb}$ of transcription start, rank 3 were within $2 \mathrm{~kb}$ of transcription start, and rank 4 were located $>2 \mathrm{~kb}$ from transcription start. Clones containing satellite repeats or $>25 \%$ Alu sequence were designated as such[18].

To examine the relationship between tumor stage and changes in DNA methylation, samples were grouped by stage, and analysis of variance (ANOVA) was performed. 2,042 CpG-rich clones with significant differences in methylation by stage at $\mathrm{p}=0.01$ (Benjamini Hochberg False Discovery Rate (FDR) adjusted) were identified, and the list reduced to 659 clones by selection for at least a 1.5fold difference in methylation between any two stages (Additional File 3). The fold change cut-off was used in addition to statistical significance to limit the number of CpG-rich sequences to those with the largest differences.

The 659 CpG-rich clones were clustered along with disease stage using hierarchical clustering with Pearson correlation and distance measured by average linkage. The resulting dendrogram showed gains and losses of DNA methylation at specific CpG-rich clones were correlated with ovarian cancer progression from normal to stage IV disease (Figure 1a). 334 CpG-rich sequences gained methylation with disease progression, while 325 lost $\mathrm{CpG}$ methylation. The majority of CpG-rich sequences that lost methylation during cancer progression were repetitive elements (satellite and Alu sequences, Figure 1a), while those that gained methylation were primarily CpG- islands associated with gene promoters (Rank 1-4, Figure 1a). A graph of the $659 \mathrm{CpG}$-rich clones by stage showed that unlike the methylated sequences, loss of methylation was not incremental with each stage progression. While stage I tumors had lost methylation relative to normal and LMP samples, the same sequences were partially methylated in stage II tumors, and reverted back to a un-methylated state in stage III and IV (Figure 1b). Examination of the 659 sequences by histopathology and stage showed that the re-methylation of sequences in stage II tumors occurred in tumors of endometrial and serous histopathologies, but not mucinous tumors (Additional File 4). Overall, the results showed an association between disease stage and progressive hyper-methylation of promoter-associated CpG islands and hypo-methylation of repetitive sequences. The same association was seen with disease grade (Additional File 5).

\section{Classification of normal or LMP and stage III samples using CPG methylation data}

The majority of ovarian tumors are stage III at the time of diagnosis. Based on the sample cluster results, we tested whether the cumulative CpG methylation changes could distinguish between normal or LMP ovarian tissue and stage III cancer. The normal, LMP, and stage III samples were randomly and evenly divided into two groups. The first group of 43 samples was used as a training set to develop a classifier of CpG-rich sequences to differentiate normal or LMP from stage III samples. The second group consisting of 44 samples was used to test the performance of the classifier on an independent data set. The Prediction Analysis of Microarrays (PAM) algorithm as implemented in BRB-ArrayTools was used to develop a classifier using all CpG-rich sequences on the microarray. The PAM algorithm optimizes the size of the classifier such that the fewest number of elements are included without decreasing performance.

The PAM algorithm produced a classifier at a threshold value of 6.26 that consisted of three CpG-rich clones, all of which were hypomethylated in stage III tumors relative to normal or LMP tissue (Figure 2). The three hypomethylated sequences included two satellite sequences and one Alu repeat (clone IDs: BF.44.F11, BF.44.B7, BF.21.B1; average methylation ratios for all clones are in Additional File 6), and all three were present in the 659 sequences identified above as significant by disease stage. During cross-validation of the classifier on the training set, the three-sequence classifier had 94\% sensitivity and 96\% specificity. One LMP and one stage III sample were incorrectly classified (sample IDs 6 and 69, Additional File 2). Overall, the classifier misclassification rate was 5\% (Figure 3). On the independent test set of samples the threesequence classifier had a sensitivity of $87 \%$ and specificity of $100 \%$ - two normal and two LMP samples were mis- 

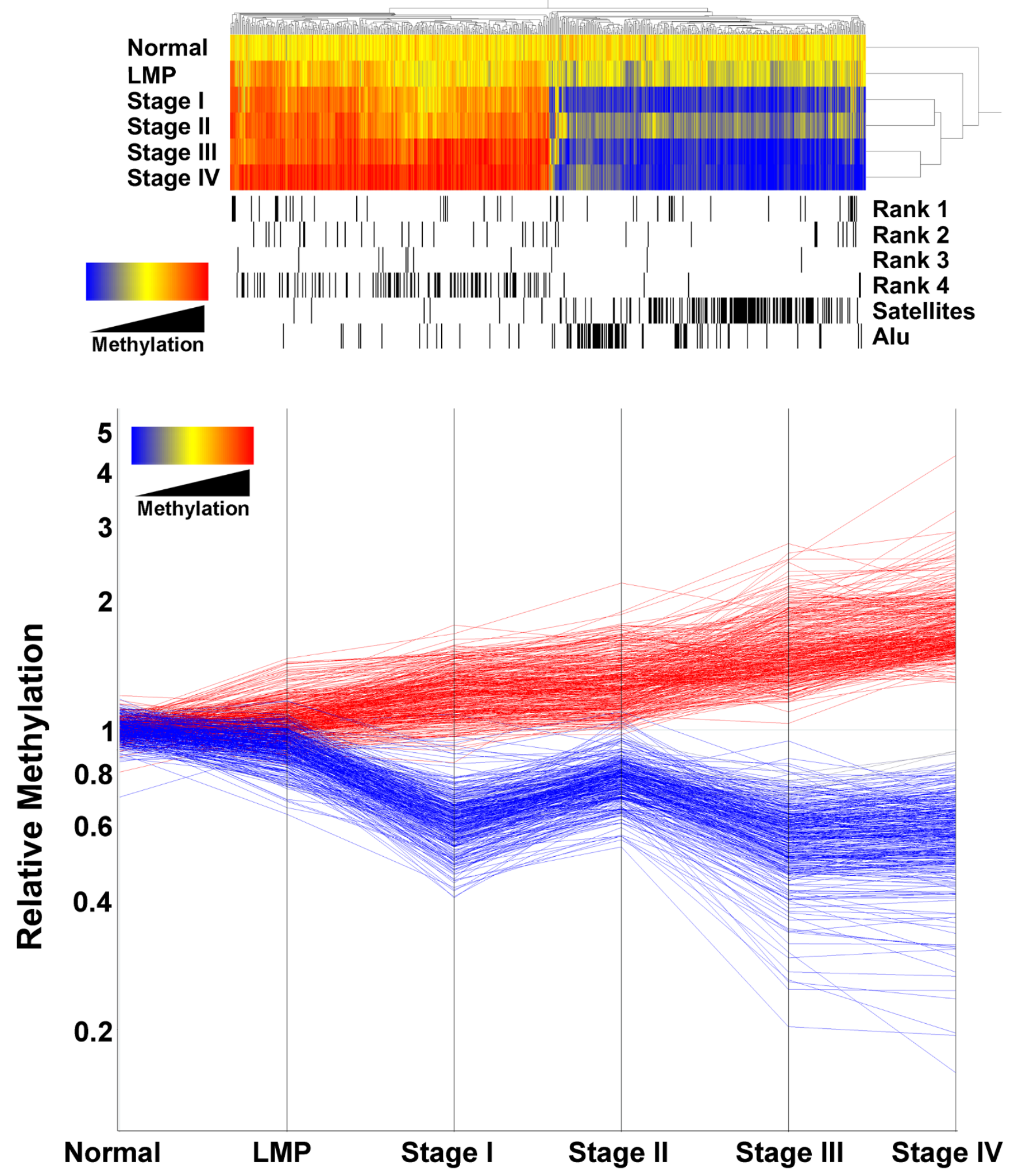

Figure I (see legend on next page) 
Figure I (see previous page)

Differences in DNA methylation patterns across the genome distinguish ovarian tissues by stage (see AdditionalFiles2and3for sample histopathology and the list of sequences used in clustering). a) The 659 CpG-rich clones with differential methylation between any two tumor stages were used in hierarchical clustering by Pearson correlation and average linkage. Each CpG-rich clone is represented by one bar and colored by their average methylation relative to the median of the ten normal samples; hypermethylation is shown in red and hypomethylation shown in blue. Horizontally aligned black bands mark the position of specific ranks of clones: Rank I, the clone sequence lies within 0.5 kb of the transcription start of a known gene; Rank 2, the clone lies within I kb of transcription start; Rank 3, the clone lies within 2 kb of transcription start; Rank 4, the clone lies $>2$ kb from transcription start; Satellites, probes containing satellite repeats; Alu, probes of which the clones consist of $>25 \%$ Alu sequence. b) The same 659 CpG-rich clones are graphed by tumor stage. Each CpG-rich clone is represented by one line. Lines are colored by their average methylation in Stage IV; blue indicates loss of methylation, and red indicates gain of methylation, relative to the median of the ten normal samples.

classified as stage III (sample IDs 62, 64, 72, 77, Additional File 2).

A separate class prediction analysis was performed in which the training and test sets were analyzed using several class prediction methods implemented in BRB ArrayTools. The training set was used to select sequences that were differentially methylated between the classes at the $p$ $<0.001$ significance level, and resulted in a classifier with 911 sequences. The 911-sequence classifier was then tested using five class prediction methods: compound covariate predictor, diagonal linear discriminant analysis, 1-nearest neighbor, 3-nearest neighbors, and support vector machines. Cross-validation using the leave-one-out method was performed to determine the mis-classification error rate of each class prediction method. To prevent bias in estimation of the mis-classification error rate, the entire analysis was repeated during each iteration of the

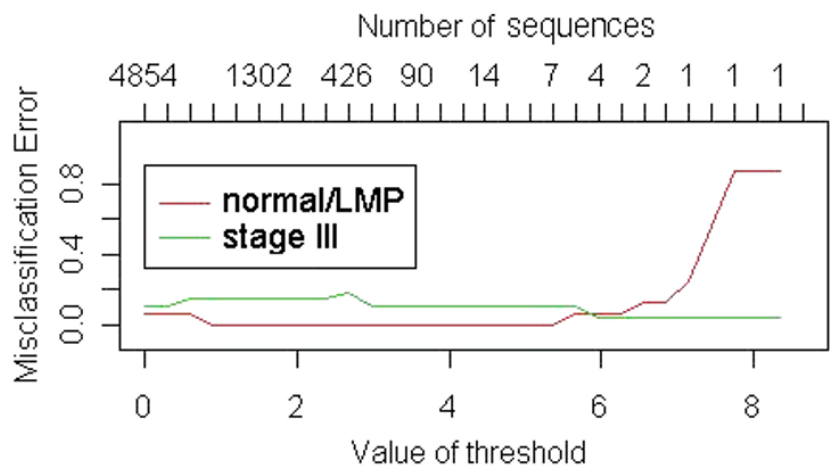

\section{Figure 2}

The optimal number of sequences to predict normal or LMP ovarian tissue from stage III ovarian tumor. Graph of misclassification rate for each class (normal/LMP tissue or stage III ovarian tumor) as a function of the threshold value in the PAM algorithm. As the threshold parameter increases, the number of sequences in the classifier decreases. The optimal point was reached at a threshold value of 6.26 , or 3 sequences. cross-validation, including determination of which sequences were significant on the reduced training sample. The sensitivity and specificity of the class prediction methods in cross-validation were nearly identical: $94 \%$ and $89 \%$ respectively for the compound covariate predictor, diagonal linear discriminant analysis, and 1-nearest neighbor methods; and $94 \%$ and $93 \%$ for the 3-nearest neighbors and support vector machines methods (Additional File 7). The class prediction methods were further validated by estimation of the permutation $\mathrm{p}$-value of the cross-validated mis-classification rate. The entire analysis was repeated 2,000 times with scrambled sample labels to determine the proportion of the random permutations that gave as small a cross-validated misclassification rate as was obtained with the real class labels. Results showed all five class prediction methods had a permutation $\mathrm{p}$ value for the cross-validated mis-classification rate of $\mathrm{p}<$ 0.001 . Finally, strong additional support for the classifier came from the independent test set of data. When used to predict the class of the samples in the independent test set, three of the class prediction methods (1-nearest neighbor, 3-nearest neighbors, and support vector machines) were $100 \%$ correct, while the remaining two methods were $98 \%$ correct; the compound covariate predictor and diagonal linear discriminant analysis methods both mis-classified sample 73 as Stage III when in fact it was a LMP sample (Additional File 7).

Of the 911 sequences with significant $(\mathrm{p}<0.001)$ methylation differences between the normal and LMP samples and the Stage III cancers, 373 had a greater than 1.5-fold change (Additional File 8). The 911 sequences used by the class prediction methods included the three sequences in the classifier developed by the PAM algorithm as well as 425 of the 659 (64\%) of the sequences identified above as significant by stage with a 1.5 -fold change in methylation. The large difference in the number (911) of sequences with significant differences between the classes and the three sequences in the classifier developed by the PAM algorithms reflected the fact that while a large number of sequences had altered CpG methylation between normal 


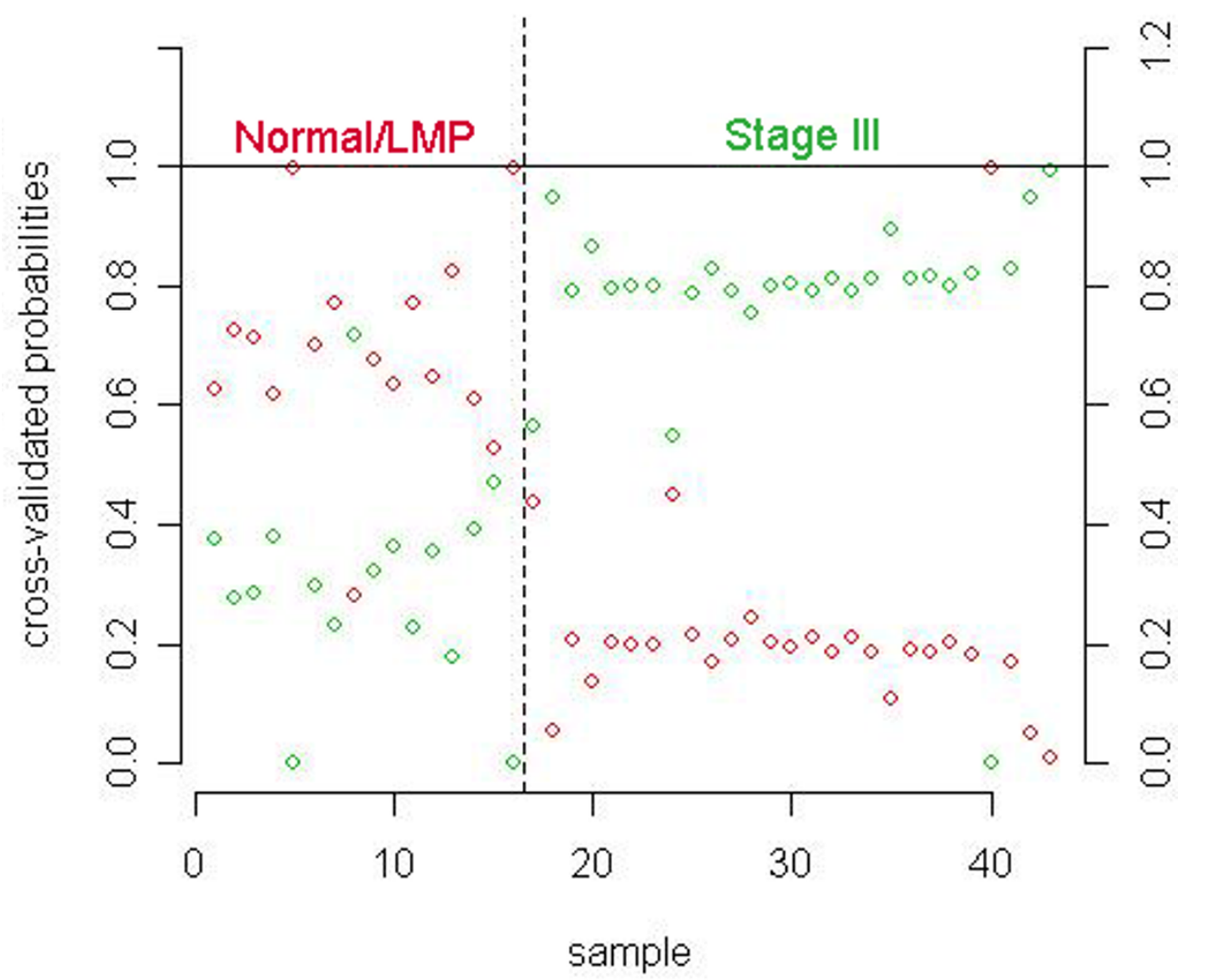

Figure 3

Performance of the 3-sequence classifier during cross-validation. The probability of each sample belonging to one class (normal or LMP tissue, red; stage III ovarian tumor, green) is shown on the y-axis for each of the 43 samples used to develop the 3-sequence classifier. One normal sample and one stage III ovarian tumor were misclassified resulting in a misclassification rate of $5 \%$.

or LMP tissue and stage III tumor (compare normal and LMP to Stage III in Figure 1), the optimal number of sequences for good performance in class prediction was small.

\section{Bisulfite sequencing confirmation of CGI microarray data} The clones with significant differences in methylation between ovarian tissues included a gene of interest: NKX23. NKX2-3 is a member of the homeodomain-containing transcription factor family, and The NKX family members have been implicated in cell type-specific gene expression and regulation of cell differentiation. Our previous work identified another family of homeobox binding proteins, the HOXA cluster, as epigenetically silenced in breast cancer[25]. We therefore examined NKX2-3 further to verify our microarray results. To confirm the methylation changes found using the CGI microarrays, we measured methylation of the NKX2-3 CpG island that overlapped the clone on the CGI microarray by bisulfite sequencing. Figure 4 a shows the position of the sequence queried on the CGI microarray relative to the region that was bisulfite sequenced, and the NKX2-3 gene sequence. Forty-seven bisulfite modified clones were sequenced from five stage III or IV tumors and five normal ovaries. The number of methylated CpG sites in each sample was counted and the result shown as a boxplot in Figure $4 \mathrm{~b}$. The difference in methylation detected between normal and tumor ovarian tissue by bisulfite sequencing was significant $(\mathrm{p}<0.01$, unpaired Welch's t-test).

\section{Re-expression of NKX2-3 by 5'-aza-deoxycytidine}

The increased methylation of the NKX2.3 CpG island suggested epigenetic silencing of NKX2-3 expression that could be reversed by the demethlyating agent 5-aza-2'deoxycytidine. To test this possibility, the ovarian cancer cell lines ES2 and OVCAR3 were treated with 5-aza-2'deoxycytidine and re-expression of the NKX2-3 gene was measured by real time RT-PCR. As expected for an epigenetically silenced gene promoter, the 5-aza-2'-deoxycytidine treatment increased expression of NKX2-3 in a dosedependent manner in both cell lines. NKX2-3 expression at $10 \mathrm{uM}$ 5-aza-2'-deoxycytidine increased 7.2-fold in ES2 

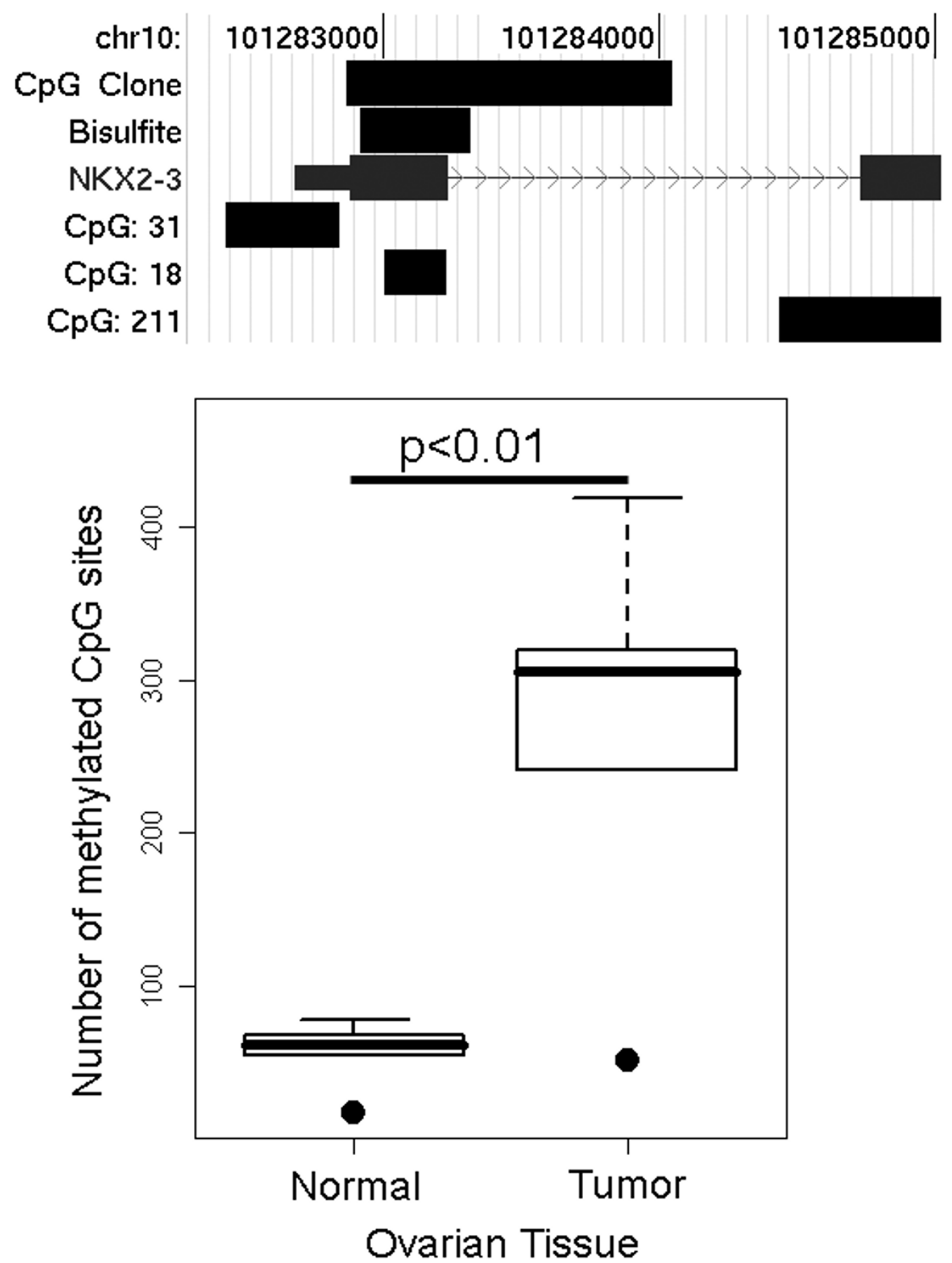

Figure 4

Confirmation of increased methylation at a NKX2-3 gene CpG island in ovarian tumor by bisulfite sequencing. a) Representation the 5' NKZ2-3 gene region showing the chromosomal location, associated CPG islands, region covered by the clone on the CGI array, region that was bisulfite sequenced, and the NKX2-3 gene itself. For the row labeled NKX2.3, the $5^{\prime}$ untranslated region is indicated by the thin bar, the first and second exons are shown as thick bars, and the first intron is indicated by a thin line with overlaid arrow heads. b) Boxplot of the bisulfite sequencing results. The total number of methylated $C_{P G}$ sites in 47 clones from each of ten samples were averaged and plotted. The $p$-value for significance between the two means is shown (unpaired Welch's t-test). 
cells while the increase in OVCAR3 cells was 359-fold (Table 1).

\section{Correlation of CPG methylation with gene expression}

Methylation of $\mathrm{CpG}$ islands associated with $5^{\prime}$ gene promoters is associated with loss of expression, as was the case with NKX2-3 above. We therefore merged the CGI array data from stage III and IV ovarian tumors with gene expression data taken from a study by Hendrix et al. [26]. In their expression study, Hendrix et al. analyzed 99 individual ovarian tumors: 35 stage I, 11 stage II, 44 stage III, 9 stage IV, and 4 normal ovary samples on Affymetrix HG_U133A GeneChips (gene expression omnibus accession number: GSE6008). Of the 659 CpG-rich clones with DNA methylation changes in our CGI array data, 201 could be mapped to the U133A GeneChip using gene names: 126 were hyper-methylated with disease progression and 75 were hypo-methylated. Further selecting for the CpG islands most closely associated with 5' promoters (rank 1 or 2) resulted in 11 hypermethylated clones and 13 hypomethylated. Of the 11 clones with increased methylation of their CpG islands, four: FLJ14146, FYCO1, TUBA3, ZNF177 were expressed in normal ovary tissue and the expression of all four decreased with disease as would be predicted from the CpG methylation profile (Figure 5a). Of the 13 clones that lost methylation with disease progression, three were expressed in Stage III and IV tumors at higher levels than in normal ovary: AHCYL1, BYSL, and DUSP10 as suggested by their CpG methylation profiles (Figure 5b).

\section{Discussion}

Genome-wide demethylation has long been thought to play a role in tumorigenesis[3]. Demethylation of the tumor cell genome has been observed in colon tumor cell lines and associated with chromosome instability in mouse stem cells $[27,28]$. The results reported here show both CpG methylation and demethylation occur with tumor progression in ovarian cancer. Demethylation occurred predominantly at repetitive elements (satellite and Alu repeats) of the genome. Demethylation of repetitive elements may contribute to genome instability and play a role in ovarian tumor progression[11]. The loss of methylation with disease progression showed a reversal between stages I and II, followed by a resumption of the trend in stage III in endometrial and serous, but not muci- nous ovarian cancer. In addition to progressive loss of methylation at repetitive elements, our results show CpG methylation increased at hundreds of promoter $\mathrm{CpG}$ islands even as the genome was becoming demethylated on average.

The results suggest that the process of tumorigenesis differentiates between types of CpG-rich elements in order to simultaneously destabilize the genome while silencing tumor suppressor genes. In addition, the cumulative nature of the CpG methylation changes suggests that the stages of ovarian cancer are progressive with one arising from another. The exception to this observation was the histopathology-specific re-methylation of Alu and satellite $\mathrm{CpG}$ islands in stage II tumors relative to stage I tumors which reverted back to a de-methylated state in stage III tumors (Additional File 4). The observation raises the possibility that either stage II tumors are unique in their formation, or that there are two waves of demethylation, from benign to stage $\mathrm{I}$, and then again in progression from stage II to stage III, during tumor progression.

To investigate the possible use of methylation patterns to identify ovarian cancer, we focused on comparing stage III serous papillary adenocarcinoma to normal and LMP tissue. Our results showed large numbers of $\mathrm{CpG}$ islands are hyper- and hypomethylated in stage III ovarian tumors relative to normal or LMP samples (Additional File 8). We were able to differentiate normal and LMP from stage III with a three-sequence classifier developed using the PAM algorithm with $87 \%$ sensitivity and $100 \%$ specificity on an independent test set of data (Figure 3). In addition, we used five other class prediction methods which had a high degree of agreement in both cross-validation of the training data set and prediction of the test data set $(98-100 \%$ correct). The agreement between the various class prediction methods supports the likelihood that our methylation data could produce a classifier capable of differentiating between Normal and LMP samples and Stage III cancer. Furthermore, estimation of the cross-validated mis-classification error rate indicated that the classifier's performance was unlikely due to chance ( $\mathrm{p}<$ $0.001)$.

The progressive nature of the methylation changes seen between stage III and normal or LMP samples suggests

Table I: 5'-aza-deoxycytidine induced re-activation of NK2-3 gene expression in ovarian cancer cell lines

\begin{tabular}{ccc}
\hline cell line & dose 5 '-aza-dC (uM) & fold increase over untreated control cells \\
\hline ES2 & 2 & 1.3 \\
ES2 & 10 & 7.2 \\
OVCAR3 & 2 & 55 \\
OVCAR3 & 10 & 359 \\
\hline
\end{tabular}



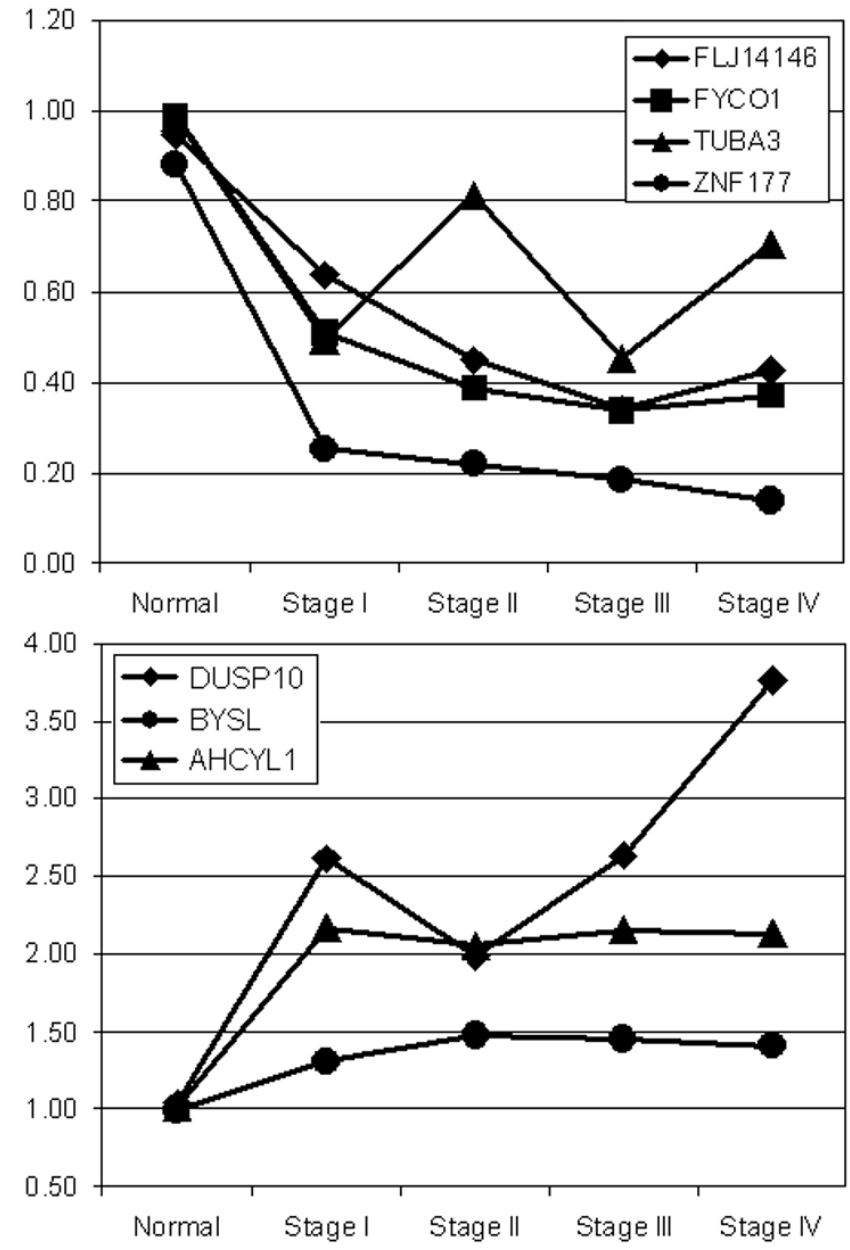

Figure 5

Expression of genes with altered expression and CpG island methylation across ovarian cancer progression. a) Expression profile of genes with increased $C_{p G}$ island methylation. b) Expression profile of genes with decreased CpG island methylation. Expression is graphed relative to the median of 4 normal samples and shown for all four stages of ovarian cancer.

that the same changes, though smaller in magnitude, may be useful in detecting early (stage I) cancers using more sensitive methods for measuring altered CpG methylation. Early detection of ovarian cancer should improve patient treatment, and is the focus of current research using protein detection in blood samples[29]. Further development of the methylation profiles identified here may provide an additional variable that could complement protein expression to further improve detection of early cancer. The demonstration that mutations in tumor DNA are detectable in the blood supports the possibility of detecting methylation events in the blood of women with ovarian cancer in order to achieve an earlier diagno$\operatorname{sis}[30]$.

To validate the CGI microarray results, we confirmed hypermethylation at a NKX2-3 CpG island in ovarian tumor samples relative to normal tissue using bisulfite sequencing. In addition, NKX2-3, was reactivated in two ovarian tumor cell lines following treatment with the demethlyating agent 5'-aza-2'deoxycytidine. Although we did not measure the methylation status of the NKX2-3 $\mathrm{CpG}$ island in the cell lines, re-expression of the gene following treatment with 5'-aza-2'deoxycytidine and confirmation of methylation in patient samples suggests that NKX2-3 is epigenetically silenced during ovarian tumorigenesis. As a further confirmation of the $\mathrm{CpG}$ island microarrays, we identified seven clones whose methylation profile predicted their loss or gain in expression with disease progression by analyzing our methylation data in conjunction with Wu et al.'s expression data. These results demonstrate that $\mathrm{CpG}$ island microarrays can be used to identify novel targets of epigenetic control in tumor samples.

\section{Conclusion}

We found patterns of DNA methylation that distinguish tumor samples from benign tissue, and used this data to discover genes affected by epigenetic regulation. We showed that the changes in methylation are cumulative with increasing stage, thus the methylation changes may provide a marker for early detection of disease. Our results extend previous studies that suggested simultaneous hypo- and hyper-methylation occurred with tumor progression by measuring both in the same tumor cells.

\section{Abbreviations}

LMP: low malignant potential; FDR: False Discovery Rate; ANOVA: analysis of variance; PAM: prediction analysis of microarrays; SVM: support vector machines.

\section{Competing interests}

There are no financial competing interests to disclose. The authors state that no financial support has been provided by any organization that stands to gain or lose financially from publication of the work presented here. The authors do not hold stock in any organization that may benefit from this publication, nor are any patents relating to this work held or applied for.

\section{Authors' contributions}

GSW: experiment conception and design, data analysis, manuscript preparation; BWF: experiment conception and design, data analysis; NH: data generation; KDG: experiment design and critical discussion, samples; FED: experiment conception and design, data analysis; SR: crit- 
ical discussion, experiment conception and design, data analysis, samples.

\section{Additional material}

\section{Additional file 1}

Three $M$ versus $A$ plots (intensity ratios $M=(R / G)$ versus average intensities $\mathrm{A}=\left(\mathrm{R}^{*} \mathrm{G}\right) / 2$ for three representative hybridizations. The sample ID is shown in the upper right of each graph.

Click here for file

[http://www.biomedcentral.com/content/supplementary/17558794-1-47-S1.tiff]

\section{Additional file 2}

Table listing all samples with their stage, histopathology, and grade. Click here for file

[http://www.biomedcentral.com/content/supplementary/17558794-1-47-S2.csv]

\section{Additional file 3}

The 659 CpG-rich clones significant ( $p<0.01,1.5$-fold change) methylation differences between any stage. All data normalized to the median of the 10 normal ovarian tissue samples. Average methylation for each stage is shown. Clones associated with a gene (rank 1-4) have the gene's accession number listed.

Click here for file

[http://www.biomedcentral.com/content/supplementary/17558794-1-47-S3.csv]

\section{Additional file 4}

The cumulative loss of DNA methylation with ovarian cancer progression reverts temporarily in endometroid and serous papillary adenocarcinomas, but not mucinous adenocarcinomas. The 659 CpG-rich clones with significant ( $p<0.01,1.5$-fold change) methylation differences between any two stages are graphed by histopathology and tumor stage. A reversion to a more normal methylation state can be seen for the sequences with overall loss of methylation in the progression from stage I to stage II in the endometroid and serous papillary adenocarcinomas. Each $C p G$-rich clone is represented by one line. Lines are colored by their average methylation in Stage IV papillary serous adenocarcinoma relative to the median of the ten normal samples; blue indicates loss of methylation, and red indicates gain of methylation.

Click here for file

[http://www.biomedcentral.com/content/supplementary/1755-

8794-1-47-S4.tiff]

\section{Additional file 5}

Cumulative loss and gain of DNA methylation in the progression from low to high grade ovarian cancer. The $659 \mathrm{Cp} G$-rich clones with significant ( $p<0.01,1.5$-fold change) changes in methylation between any two stages of cancer are graphed by grade. Each $C p G$-rich clone is represented by one line. Lines are colored by their average methylation in grade 3 tumors relative to the median of the ten normal samples; blue indicates loss of methylation, and red indicates gain of methylation. The average methylation of the normal and low malignant potential samples is shown combined as "benign".

Click here for file

[http://www.biomedcentral.com/content/supplementary/17558794-1-47-S5.tiff]

\section{Additional file 6}

Data for all CpG-rich clones on the CGI microarray with replicates spots averaged. All data normalized to the median of the 10 normal ovarian tissue samples. Average methylation for each stage is shown. Clones associated with a gene (rank 1-4) have the gene's accession number listed.

Click here for file

[http://www.biomedcentral.com/content/supplementary/17558794-1-47-S6.csv]

\section{Additional file 7}

The results of the BRB ArrayTools analysis are shown, including crossvalidation results, and results on the test for each of the five class prediction methods, as well as the list of the 911 sequences that compose the classifier. An additional sheet shows the methylation data for the 911 sequences across all disease stages with all data normalized to the median of the ten normal ovarian tissue samples. Average methylation for each stage is shown. Clones associated with a gene (rank 1-4) have the gene's accession number listed.

Click here for file

[http://www.biomedcentral.com/content/supplementary/17558794-1-47-S7.xls]

\section{Additional file 8}

The 373 CpG-rich clones used by the class prediction methods with 1.5-fold changes in DNA methylation between normal and LMP, and stage III samples graphed by tumor stage. Each CpG-rich clone is represented by one line. Lines are colored by their average methylation in Stage IV relative to the median of the ten normal samples; blue indicates loss of methylation, and red indicates gain of methylation.

Click here for file

[http://www.biomedcentral.com/content/supplementary/17558794-1-47-S8.tiff]

\section{Acknowledgements}

Class prediction analyses were performed using BRB-ArrayTools Version 3.5.0 developed by Dr. Richard Simon and Amy Peng Lam. Microarray analysis was performed with assistance from the Genomics Shared Service at the Arizona Cancer Center supported by NIEHS grant ES06694, NIH grant CA23074, and the BIO5 institute. Sequencing of bisulfite modified DNA clones was performed by the Genomic Analysis, Technology, \& Sequencing Core at the University of Arizona. The authors wish to thank Dr. Molly Brewer, University of Connecticut, for helpful discussions. This research was supported by NIH ROI CA736 I 2 to F.E.D. and NIH R33 CA09I35I to B.W.F.

\section{References}

I. American Cancer Society: Cancer facts \& figures. Atlanta, GA: The Society; 2007.

2. Jaenisch R, Bird A: Epigenetic regulation of gene expression: how the genome integrates intrinsic and environmental signals. Nat Genet 2003, 33(Suppl):245-254.

3. Fearon ER, Vogelstein B: A genetic model for colorectal tumorigenesis. Cell I 990, 6 I (5):759-767.

4. Jones PA, Baylin SB: The fundamental role of epigenetic events in cancer. Nat Rev Genet 2002, 3(6):4I5-428.

5. Laird PW: Cancer epigenetics. Hum Mol Genet 2005, I 4(Spec No I):R65-76.

6. Costello JF, Fruhwald MC, Smiraglia DJ, Rush LJ, Robertson GP, Gao $X$, Wright FA, Feramisco JD, Peltomaki P, Lang JC, et al.: Aberrant CpG-island methylation has non-random and tumour-typespecific patterns. Nat Genet 2000, 24(2): 132-138. 
7. Yan PS, Perry MR, Laux DE, Asare AL, Caldwell CW, Huang TH: CpG island arrays: an application toward deciphering epigenetic signatures of breast cancer. Clin Cancer Res 2000, 6(4): $|432-| 438$.

8. Zardo G, Tiirikainen MI, Hong C, Misra A, Feuerstein BG, Volik S, Collins CC, Lamborn KR, Bollen A, Pinkel D, et al:: Integrated genomic and epigenomic analyses pinpoint biallelic gene inactivation in tumors. Nat Genet 2002, 32(3):453-458.

9. Shi H, Wei SH, Leu YW, Rahmatpanah F, Liu JC, Yan PS, Nephew KP, Huang TH: Triple analysis of the cancer epigenome: an integrated microarray system for assessing gene expression, DNA methylation, and histone acetylation. Cancer Res 2003, 63(9):2164-2I7I.

10. Wei SH, Balch C, Paik HH, Kim YS, Baldwin RL, Liyanarachchi S, Li L, Wang Z, Wan JC, Davuluri RV, et al.: Prognostic DNA methylation biomarkers in ovarian cancer. Clin Cancer Res 2006, I 2(9):2788-2794.

II. Widschwendter M, Jiang G, Woods C, Muller HM, Fiegl H, Goebel G Marth C, Muller-Holzner E, Zeimet AG, Laird PW, et al.: DNA hypomethylation and ovarian cancer biology. Cancer Res 2004, 64(I3):4472-4480.

12. Kunze E, Wendt M, Schlott T: Promoter hypermethylation of the 14-3-3 sigma, SYK and CAGE-I genes is related to the various phenotypes of urinary bladder carcinomas and associated with progression of transitional cell carcinomas. Int J Mol Med 2006, 18(4):547-557.

13. Mori T, Martinez SR, O'Day SJ, Morton DL, Umetani N, Kitago M, Tanemura A, Nguyen SL, Tran AN, Wang HJ, et al.: Estrogen receptor-alpha methylation predicts melanoma progression. Cancer Res 2006, 66(13):6692-6698.

14. Oue N, Mitani Y, Motoshita J, Matsumura S, Yoshida K, Kuniyasu H, Nakayama $\mathrm{H}$, Yasui W: Accumulation of DNA methylation is associated with tumor stage in gastric cancer. Cancer 2006, 106(6): 1250-1259.

15. Bastian PJ, Ellinger J, Wellmann A, Wernert N, Heukamp LC, Muller $S C$, von Ruecker A: Diagnostic and prognostic information in prostate cancer with the help of a small set of hypermethylated gene loci. Clin Cancer Res 2005, I I (I I):4097-4I06.

16. Christoph F, Weikert S, Kempkensteffen C, Krause H, Schostak M, Kollermann J, Miller K, Schrader M: Promoter hypermethylation profile of kidney cancer with new proapoptotic p53 target genes and clinical implications. Clin Cancer Res 2006, I 2( I 7):5040-5046.

17. Sambrook J, Russell DW: Molecular cloning: a laboratory manual. 3rd edition. Cold Spring Harbor, N.Y.: Cold Spring Harbor Laboratory Press; $200 \mathrm{I}$.

18. Nouzova M, Holtan N, Oshiro MM, Isett RB, Munoz-Rodriguez JL, List AF, Narro ML, Miller SJ, Merchant NC, Futscher BW: Epigenomic changes during leukemia cell differentiation: analysis of histone acetylation and cytosine methylation using CPG island microarrays. J Pharmacol Exp Ther 2004, 3 I I (3):968-98 I.

19. Finkelstein D, Ewing R, Gollub J, Sterky F, Cherry JM, Somerville S: Microarray data quality analysis: lessons from the AFGC project. Arabidopsis Functional Genomics Consortium. Plant Mol Biol 2002, 48(I-2): I I9-I3I.

20. Groot GS, Kroon AM: Mitochondrial DNA from various organisms does not contain internally methylated cytosine in CCGG- sequences. Biochim Biophys Acta 1979, 564(2):355-357.

21. Simon R, Radmacher MD, Dobbin K, McShane LM: Pitfalls in the use of DNA microarray data for diagnostic and prognostic classification. J Natl Cancer Inst 2003, 95(I): 14-I8.

22. Clark SJ, Harrison J, Paul CL, Frommer M: High sensitivity mapping of methylated cytosines. Nucleic Acids Res 1994, 22(I5):2990-2997.

23. Watts GS, Oshiro MM, Junk DJ, Wozniak RJ, Watterson S, Domann FE, Futscher BW: The acetyltransferase p300/CBP-associated factor is a p53 target gene in breast tumor cells. Neoplasia 2004, 6(3): 187-194

24. Antequera $F$, Bird $A$ : Number of $C p G$ islands and genes in human and mouse. Proc Natl Acad Sci USA 1993. 90(24): I I 995-1 I 999.

25. Novak P, Jensen T, Oshiro MM, Wozniak RJ, Nouzova M, Watts GS, Klimecki WT, Kim C, Futscher BW: Epigenetic inactivation of the HOXA gene cluster in breast cancer. Cancer Res 2006, 66(22): 10664-10670.
26. Hendrix ND, Wu R, Kuick R, Schwartz DR, Fearon ER, Cho KR: Fibroblast growth factor 9 has oncogenic activity and is a downstream target of Wnt signaling in ovarian endometrioid adenocarcinomas. Cancer Res 2006, 66(3): | 354-1362.

27. Lengauer C, Kinzler KW, Vogelstein B: Genetic instability in colorectal cancers. Nature 1997, 386(6625):623-627.

28. Chen RZ, Pettersson U, Beard C, Jackson-Grusby L, Jaenisch R: DNA hypomethylation leads to elevated mutation rates. Nature 1998, 395(6697):89-93.

29. Visintin I, Feng Z, Longton G, Ward DC, Alvero AB, Lai Y, Tenthorey J, Leiser A, Flores-Saaib R, Yu H, et al:: Diagnostic markers for early detection of ovarian cancer. Clin Cancer Res 2008, I 4(4): $1065-1072$

30. Diehl F, Li M, Dressman D, He Y, Shen D, Szabo S, Diaz LA Jr, Goodman SN, David KA, Juhl H, et al.: Detection and quantification of mutations in the plasma of patients with colorectal tumors. Proc Natl Acad Sci USA 2005, 102(45): I6368- 16373.

\section{Pre-publication history}

The pre-publication history for this paper can be accessed here:

http://www.biomedcentral.com/1755-8794/1/47/prepub
Publish with Biomed Central and every scientist can read your work free of charge

"BioMed Central will be the most significant development for disseminating the results of biomedical research in our lifetime. "

Sir Paul Nurse, Cancer Research UK

Your research papers will be:

- available free of charge to the entire biomedical community

- peer reviewed and published immediately upon acceptance

- cited in PubMed and archived on PubMed Central

- yours - you keep the copyright
BioMedcentral 